\title{
Role of dissolved oxygen on the plankton dynamics in spatio-temporal domain
}

\author{
Joydip Dhar $^{1}$ (D) Randhir Singh Baghel $^{2}$
}

Received: 19 November 2015/Accepted: 23 November 2015/Published online: 19 December 2015

(C) Springer International Publishing Switzerland 2015

\begin{abstract}
A mathematical model is proposed to study the role of dissolved oxygen in the plankton ecosystem in spatiotemporal domain, considering one nonliving compartment, i.e., dissolved oxygen and two living compartments, i.e., phytoplankton and zooplankton populations with Holling type II response function for the harvesting rate of phytoplankton by zooplankton population. In temporal system, the local stability analysis of all the feasible equilibria is studied and also explored the existence of Hopf-bifurcation for the interior equilibrium, taking the growth rate of phytoplankton as bifurcation parameter. Further, the direction of Hopf-bifurcation and stability of the bifurcating periodic solutions are presented using normal form theory. In spatial system, we have obtained the condition for diffusion driven instability and obtained different types of spatial patterns with different step size in time. Furthermore, conducted the higher-order stability analysis of the spatiotemporal dynamics. Finally, numerical simulation is given in support of the analytic results for both temporal and spatiotemporal domain.
\end{abstract}

Keywords Aquatic ecosystem - Hopf bifurcation . Diffusion instability $\cdot$ Spatial patterns $\cdot$ Higher order stability

Joydip Dhar

jdhar.iiitmg@gmail.com

Randhir Singh Baghel

randhirsng@gmail.com

1 Department of Applied Sciences, ABV-Indian Institute of Information Technology and Management, Gwalior 474015, India

2 Department of Mathematics, Pratap Institute of Technology \& Science, Sikar 332001, Rajasthan, India
Mathematics Subject Classification 34C23 - 34D20 . 92B05 $\cdot 92 \mathrm{D} 40$

\section{Introduction}

Last few decade, aquatic ecologists are fascinated by the dynamics of explosive phytoplankton blooms (i.e., the rapid growth and decay in their population) (Huppert et al. 2005). The blooms are of two types, one is spring bloom and other is red bloom. The spring bloom is seasonal, it is due to change in temperature and nutrient level associated with season. On the other hand, red bloom is localized out break associated with change in water temperature and with greater stability of water column (Truscott and Brindley 1994). The classification of phytoplankton blooms are reported in literature (Agusti et al. 1987; Bratbak et al. 1995). Sometimes blooms are viewed as a signal of approaching eutrophication, indicating that nutrients may have reached at unacceptably high levels, at least high enough to support the bloom formation (Huppert et al. 2005). In reality, phytoplankton bloom is a natural event of ecosystem which is not a cause of concern or danger to water quality. In real world, frequent outcome of a planktonic bloom formation leads to massive cell lysis and rapid disintegration of large planktonic population. This is closely followed by an equally rapid increase in bacterial number and in turn, by fast deoxygenating of water, which could be detrimental to aquatic plants and animals (Sarkar and Malchow 2005). Several researchers have tried to explain the nature of bloom dynamics in planktonic system using different approaches namely: nutrient upwelling, spatial patchiness and species diversity (Edward and Brindley 1996; Mathews and Brindley 1996; Pitchford and Brindley 1998; Truscott and Brindley 1994). 
The role of different functional forms in zooplanktonphytoplankton interaction was studied (Baghel et al. 2012; Ruan 1993). During recent years, many models were proposed to study the plankton system with nutrients availability. The eutrophication has already been applied to many coastal ecosystems all over the world (Zhang et al. 2004). It has been observed that synthetic eutrophication plays a significant role for excitable behavior of the system and depends upon the ratio of nitrogen to phosphorous (Egg and Heimdal 1994). Researchers have suggested that eutrophication resulting from human activities has led to an increase in phytoplankton production in Kattegat throughout the annual period in which nutrients are favorable for the growth of phytoplankton (Baghel et al. 2011, 2012; Richardson and Heilmann 1995).

In spatial ecology, the fundamental importance of patterns in biology is self-evident (Murray 2003). Pattern formation studies have often been criticized for their lack of inclusion of factors in the models. It should be remembered that the generation of pattern and form, particularly in development, is usually a long way from the level of the genome (Petrovskii and Malchow 2001). Many of the evolving patterns could hardly have been anticipated solely by genetic information. Much of the research in developmental biology, both experimental and theoretical, is devoted to trying to determine the underlying mechanisms which generate pattern and form in early development (Cantrell and Cosner 2004; Murray 2003).

It is well known natural phenomenon that a substance goes from high density regions to low density regions and hence spatial patterns are formed in nature. Our understanding is such that whatever pattern, observe in the animal world, it is almost certain that the process that produced it is still unknown. Moreover, the spatial element of ecological interactions have been identified as an crucial factor in how ecological communities are shaped (Medvinsky et al. 2002; Murray 2003; Petrovskii and Malchow 1999; Yi et al. 2009). Recently, few articles on plankton dynamics in spatiotemporal domain with instant nutrient replenishment in closed environment have been published (Baghel et al. 2011; Dhar et al. 2012) and the disease class phytoplankton with three dimensional pattern formation is also obtained (Baghel et al. 2012).

Several investigators have studied the dissolve oxygen on the existence of interacting planktonic population (Khare and Kumar 2013; Naik and Manjapp 2010) and also many authors have analyzed depletion of dissolved oxygen in eutrophied water bodies (Misra 2010; Shuklaa and Misrac 2008).

Keeping in view the above discussion, we have proposed a mathematical model to study the role of dissolved oxygen on the plankton dynamics and its schematic flow is shown in Fig. 1, where $P, Z$ and $D$ are respectively

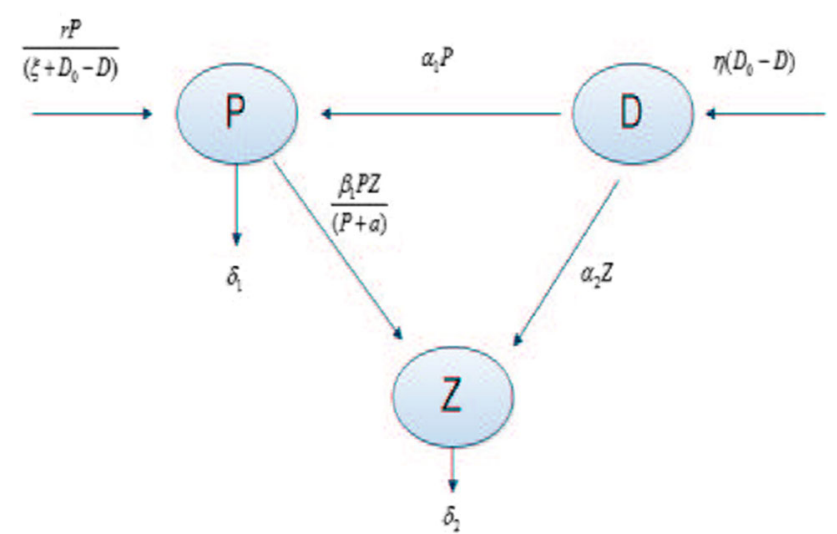

Fig. 1 Schematic diagram of proposed model

densities of phytoplankton, zooplankton population and concentration of dissolved oxygen in water.

Our main intension in this paper is to analyze the proposed mathematical models in both temporal and spatiotemporal domain. The rest of this paper is organized as follows: In "Formulation of mathematical model", we have proposed temporal model and studied the local stability and existence of Hopf-bifurcation with direction and stability of Hopf-bifurcating periodic solution. In "Model with diffusion", we have proposed model with diffusion, analyzed the diffusion instability and also carried out numerical simulation in order to obtain the different types of spatial pattern. In "Higher order stability analysis", we have conducted the higher order stability analysis for spatiotemporal system. Finally, last section "Conclusions" is given.

\section{Formulation of mathematical model}

Keeping in view the above, we consider $P(t)$ and $Z(t)$ are representation of densities of phytoplankton and zooplankton population respectively at any time $t$, and the interaction among the phytoplankton and zooplankton with Holling type-II functional response, because initially phytoplankton is more vulnerable to predation by zooplankton till the population density of phytoplankton reaches certain threshold value. Let $D(t)$ stands for concentration of dissolved oxygen in water at any time $t$. Further, $D_{0}$ is the constant intake of dissolved oxygen in water. The intrinsic growth rate of phytoplankton increase with respect to $D$ and suppose that the maximum growth rate is $r / \zeta$ at $D=D_{0}$. Then, we can consider the growth rate function of phytoplankton due to the uptake of dissolved oxygen as $\frac{r}{\zeta+D_{0}-D}$. Also, $\delta_{1}, \delta_{2}$ are the natural death rates of phytoplankton and zooplankton which includes the natural washout respectively. The up-taking rate of phytoplankton by zooplankton and conversion rate of phytoplankton into zooplankton are represented by $\beta$ and $\beta_{1}$ respectively. 
Again, the dissolved oxygen may not directly involve for the growth of zooplankton, but it will be utilized by them for their survival, let $\alpha_{1}, \alpha_{2}$ be the rates of uptake of dissolved oxygen by per capita phytoplankton and zooplankton respectively. We have taken $\eta$ as the rate of replenishment of oxygen in the water reservoir. The dynamical system is governed by the following set of ordinary differential equations:

$\frac{d P}{d t}=\frac{r P}{\left(\zeta+D_{0}-D\right)}-\delta_{1} P-\frac{\beta P Z}{(P+a)}$,

$\frac{d D}{d t}=\eta\left(D_{0}-D\right)-\alpha_{1} P-\alpha_{2} Z$,

$\frac{d Z}{d t}=\frac{\beta_{1} P Z}{(P+a)}-\delta_{2} Z$,

with non-negative initial conditions $P(0)>0, D(0)>0$, $Z(0)>0$. The Holling type-II functional response $\frac{P Z}{(P+a)}$ has been used earlier by many other researchers also Holling (1959).

\section{Equilibria and local stability analysis}

Now, we will study the existence of all possible steady states of the temporal system. The system (1)-(3) has three feasible equilibriums,

(i) $E_{0}=\left(0, D_{0}, 0\right)$,

(ii) $E_{1}=\left(\frac{\eta\left(r-\delta_{1} \zeta\right)}{\alpha_{1} \delta_{1}},\left(D_{0}+\zeta\right)-\frac{r}{\delta_{1}}, 0\right)$ exits when $\frac{r}{\delta_{1}}-D_{0}<\zeta<\frac{r}{\delta_{1}}$

(iii) $E_{2}=\left(P^{*}, D^{*}, Z^{*}\right)$ where $P^{*}=\frac{a \delta_{2}}{\beta_{1}-\delta_{2}}$ exits when $\beta_{1}>\delta_{2}, \quad Z^{*}=\frac{a \beta_{1}}{\beta\left(\beta_{1}-\delta_{2}\right)}\left(\frac{r}{\zeta+D_{0}-D^{*}}-\delta_{1}\right) \quad$ and $D^{*}=\left(D_{0}-\frac{a \alpha_{1} \delta_{2}}{\eta\left(\beta_{1}-\delta_{2}\right)}-\frac{\alpha_{2} Z^{*}}{\eta}\right)$.

Corresponding to the equilibrium point $E_{0}=\left(0, D_{0}, 0\right)$, Jacobian $J_{1}$ is given by

$J_{1}=\left[\begin{array}{ccc}0 & 0 & 0 \\ -\alpha_{1} & -\eta & -\alpha_{2} \\ 0 & 0 & 0\end{array}\right]$.

The Jacobian $J_{1}$ has the eigenvalues $\lambda_{1}=0, \lambda_{2}=-\eta$, $\lambda_{3}=0$.

Hence, $E_{0}$ is one directional stable and two directional unstable.

The variation matrix corresponding to the equilibrium point $E_{1}$ is given by Jacobian $J_{2}$ where

$$
J_{2}=\left[\begin{array}{ccc}
0 & \frac{\eta \delta_{1}\left(r-\delta_{1} \zeta\right)}{\alpha_{1}} & \frac{-\beta \eta\left(r-\delta_{1} \zeta\right)}{a \alpha_{1} \delta_{1}+\eta\left(r-\delta_{1} \zeta\right)} \\
-\alpha_{1} & -\eta & -\alpha_{2} \\
0 & 0 & 0
\end{array}\right] .
$$

The characteristic equation is given by

$\lambda^{3}+B_{1} \lambda^{2}+B_{2} \lambda+B_{3}=0$,

where $\quad B_{1}=\eta, \quad B_{2}=\frac{\eta \delta_{1}}{\alpha_{1}}\left(\zeta \delta_{1}-r\right), \quad B_{3}=\frac{\eta \delta_{1} \alpha}{\alpha_{1}}\left(\zeta \delta_{1}^{2}-r\right)$. Hence, using Routh-Hurwitz criteria $E_{1}$ is locally asymptotically stable, if $B_{i}>0, i=1,2,3$ and $B_{1} B_{2}>B_{3}$.

The general variational matrix corresponding to the equilibrium point $E_{2}$ is given by

$$
J^{*}=\left[\begin{array}{ccc}
\frac{\beta Z^{*}}{a+P^{*}}-\frac{a \beta Z^{*}}{\left(a+P^{*}\right)^{2}} & \frac{r P^{*}}{\left(\zeta+D_{0}-D^{*}\right)^{2}} & -\frac{\beta P^{*}}{a+P^{*}} \\
-\alpha_{1} & -\eta & -\alpha_{2} \\
\frac{a \beta_{1} Z^{*}}{\left(a+P^{*}\right)^{2}} & 0 & 0
\end{array}\right],
$$

and its characteristic equation is:

$\lambda^{3}+A_{1} \lambda^{2}+A_{2} \lambda+A_{3}=0$,

where

$A_{1}=\eta-\frac{\beta P^{*} Z^{*}}{\left(a+P^{*}\right)^{2}}$,

$A_{2}=\frac{r \alpha_{1} P^{*}}{\left(\zeta+D_{0}-D^{*}\right)^{2}}+\frac{\beta P^{*} Z^{*}}{\left(a+P^{*}\right)^{2}}\left(\frac{a \beta_{1}}{a+P^{*}}-\eta\right)$,

$A_{3}=\frac{a \beta_{1} P^{*} Z^{*}}{\left(a+P^{*}\right)^{2}}\left(\frac{r \alpha_{2}}{\left(\zeta+D_{0}-D^{*}\right)^{2}}+\frac{r \beta}{\left(a+P^{*}\right)}\right)$.

Hence, using Routh-Hurwitz criteria $E_{2}$ is locally asymptotically stable, if $A_{i}>0, i=1,2,3$ and $A_{1} A_{2}>A_{3}$.

\section{Existence of Hopf bifurcation}

In this subsection, we will study the Hopf-bifurcation of system (1)-(3), taking " $r$ " (i.e., the growth rate of phytoplankton due to the up-taking of dissolved oxygen) as the bifurcation parameter. Now, the necessary and sufficient condition for the existence of the Hopf-bifurcation, if there exists $r=r_{0}$ such that (i) $A_{i}\left(r_{0}\right)>0, i=1,2,3$, (ii) $A_{1}\left(r_{0}\right) A_{2}\left(r_{0}\right)-A_{3}\left(r_{0}\right)=0$ and (iii) if we consider the eigenvalues of the characteristic equation (4) of the form $\lambda_{i}=u_{i}+i v_{i}$, then $\operatorname{Re} \frac{d}{d r}\left(u_{i}\right) \neq 0, i=1,2,3$.

Now, we will verify the condition (iii) of Hopf-bifurcation. Put $\lambda=u+i v$ in (4), we get

$$
(u+i v)^{3}+A_{1}(u+i v)^{2}+A_{2}(u+i v)+A_{3}=0 .
$$

On separating the real and imaginary parts and eliminating $v$ between real and imaginary parts, we get

$8 u^{3}+8 A_{1} u^{2}+2\left(A_{1}^{2}+A_{2}\right) u+A_{1} A_{2}-A_{3}=0$.

Now, we have $u\left(r_{0}\right)=0$ as $A_{1}\left(r_{0}\right) A_{2}\left(r_{0}\right)-A_{3}\left(r_{0}\right)=0$. Further, $\quad r=r_{0}, \quad$ is the only positive root of $A_{1}\left(r_{0}\right) A_{2}\left(r_{0}\right)-A_{3}\left(r_{0}\right)=0$, and the discriminate of $8 u^{2}+$ 
$8 A_{1} u+2\left(A_{1}^{2}+A_{2}\right)=0$ is $-64 A_{2}<0$. Here, differentiating

(6) with respect to $r$, we have $\left(24 u^{2}+16 A_{1} u+2\left(A_{1}^{2}+\right.\right.$ $\left.\left.A_{2}\right)\right) \frac{d u}{d r}+\left(8 u^{2}+4 A_{1} u\right) \frac{d A_{1}}{d r}+2 u \frac{d A_{2}}{d r}+\frac{d}{d r}\left(A_{1} A_{2}-A_{3}\right)=0$.

Now, since at $r=r_{0}, u\left(r_{0}\right)=0$, we get $\left[\frac{d u}{d r}\right]_{r=r_{0}}=$ $\frac{-\frac{d}{d r}\left(A_{1} A_{2}-A_{3}\right)}{2\left(A_{1}^{2}+A_{2}\right)} \neq 0$, which ensures that the above system has a

Hopf-bifurcation. It is shown graphically in Figs. 2, 3.

\section{Direction and stability of the hopf bifurcation}

Let $x_{1}=P-P^{*}, y_{1}=D-D^{*}, z_{1}=Z-Z^{*}$, then put in the system (1)-(3), we can shift the equilibrium to the origin:

$$
\begin{aligned}
\frac{d x_{1}}{d t}= & \frac{r x_{1}}{\left(\zeta+D_{0}-D^{*}-y_{1}\right)}+\frac{r P^{*} y_{1}}{\left(\zeta+D_{0}-D^{*}-y_{1}\right)\left(\zeta+D_{0}-D^{*}\right)} \\
& -\frac{\beta\left(x_{1} z_{1}+x_{1} Z^{*}+P^{*} z_{1}\right)}{\left(a+P^{*}+x_{1}\right)}+\frac{\beta P^{*} Z^{*} x_{1}}{\left(a+P^{*}+x_{1}\right)\left(a+P^{*}\right)}-\delta_{1} x_{1},
\end{aligned}
$$

$\frac{d y_{1}}{d t}=-\left(\eta y_{1}+\alpha_{1} x_{1}+\alpha_{2} z_{1}\right)$, $\frac{d z_{1}}{d t}=\frac{\beta_{1}\left(x_{1} z_{1}+x_{1} Z^{*}+P^{*} z_{1}\right)}{\left(a+P^{*}+x_{1}\right)}-\frac{\beta_{1} P^{*} Z^{*} x_{1}}{\left(a+P^{*}+x_{1}\right)\left(a+P^{*}\right)}-\delta_{2} z_{1}$.

Its jacobian matrix $\mathrm{J}$ at point $E_{2}\left(P^{*}, D^{*}, Z^{*}\right)$ is

$$
J(0)=\left[\begin{array}{ccc}
\frac{\beta P^{*} Z^{*}}{\left(a+P^{*}\right)^{2}} & \frac{r P^{*}}{\left(\zeta+D_{0}-D^{*}\right)^{2}} & -\frac{\beta P^{*}}{a+P^{*}} \\
-\alpha_{1} & -\eta & -\alpha_{2} \\
\frac{a \beta_{1} Z^{*}}{\left(a+P^{*}\right)^{2}} & 0 & 0
\end{array}\right],
$$

and its corresponding characteristic equation is $\lambda^{3}+A_{1} \lambda^{2}+A_{2} \lambda+A_{3}=0$,

where $A_{1} A_{2}=A_{3}$,

$\left(\lambda^{2}+A_{2}\right)\left(\lambda+A_{1}\right)=0$.

Obviously Eq. (10) has a pair of purely imaginary conjugate roots $\lambda_{1,2}= \pm i \omega$ and a real root $\lambda_{3}=-A_{1}$ where $A_{1}=$ $\left(\beta P^{*} H-\eta\right)$ and $H=\frac{Z^{*}}{\left(a+P^{*}\right)^{2}}$.
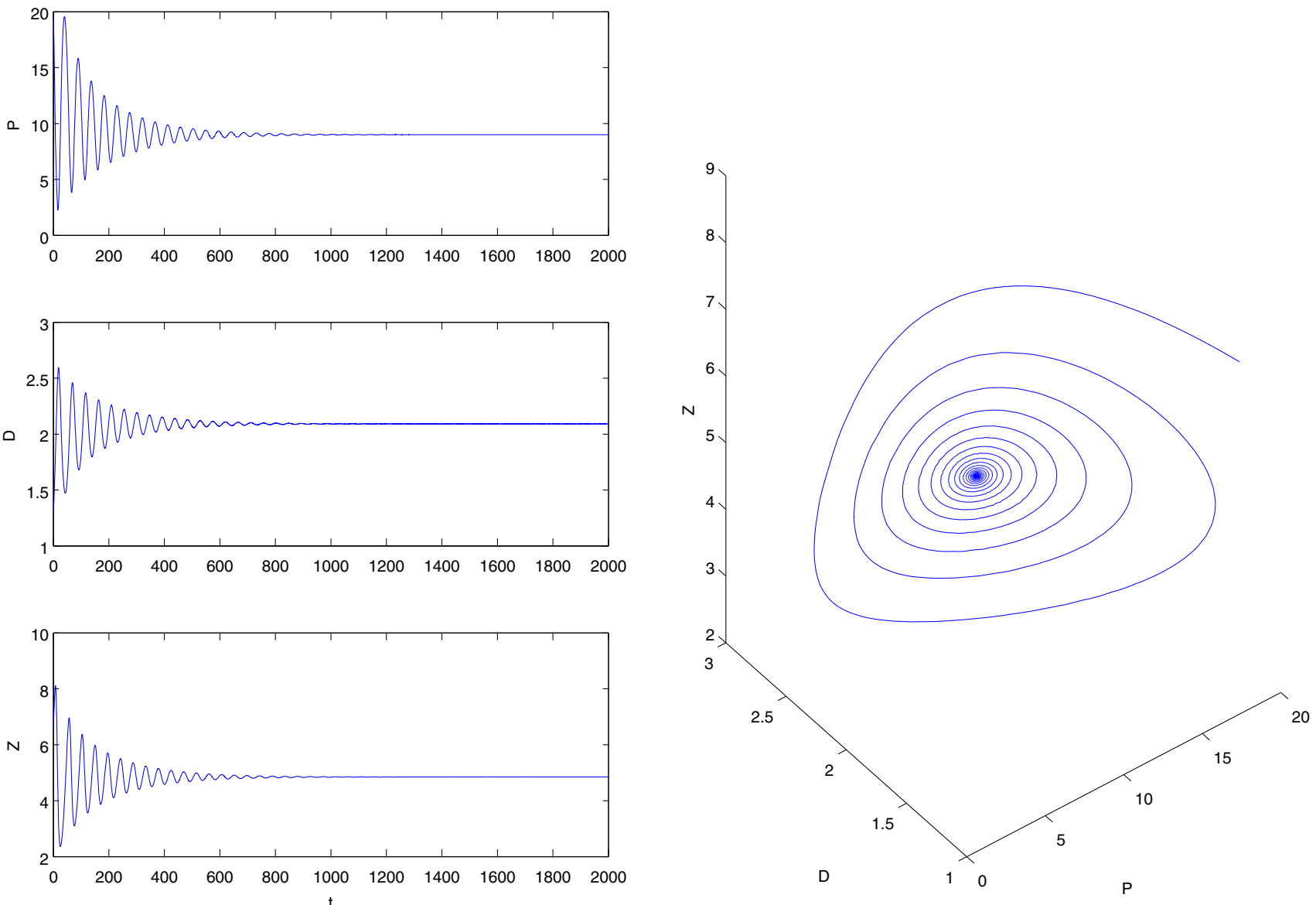

Fig. 2 The time series and phase space representation of three species around the endemic equilibrium, with $r=0.29$ 

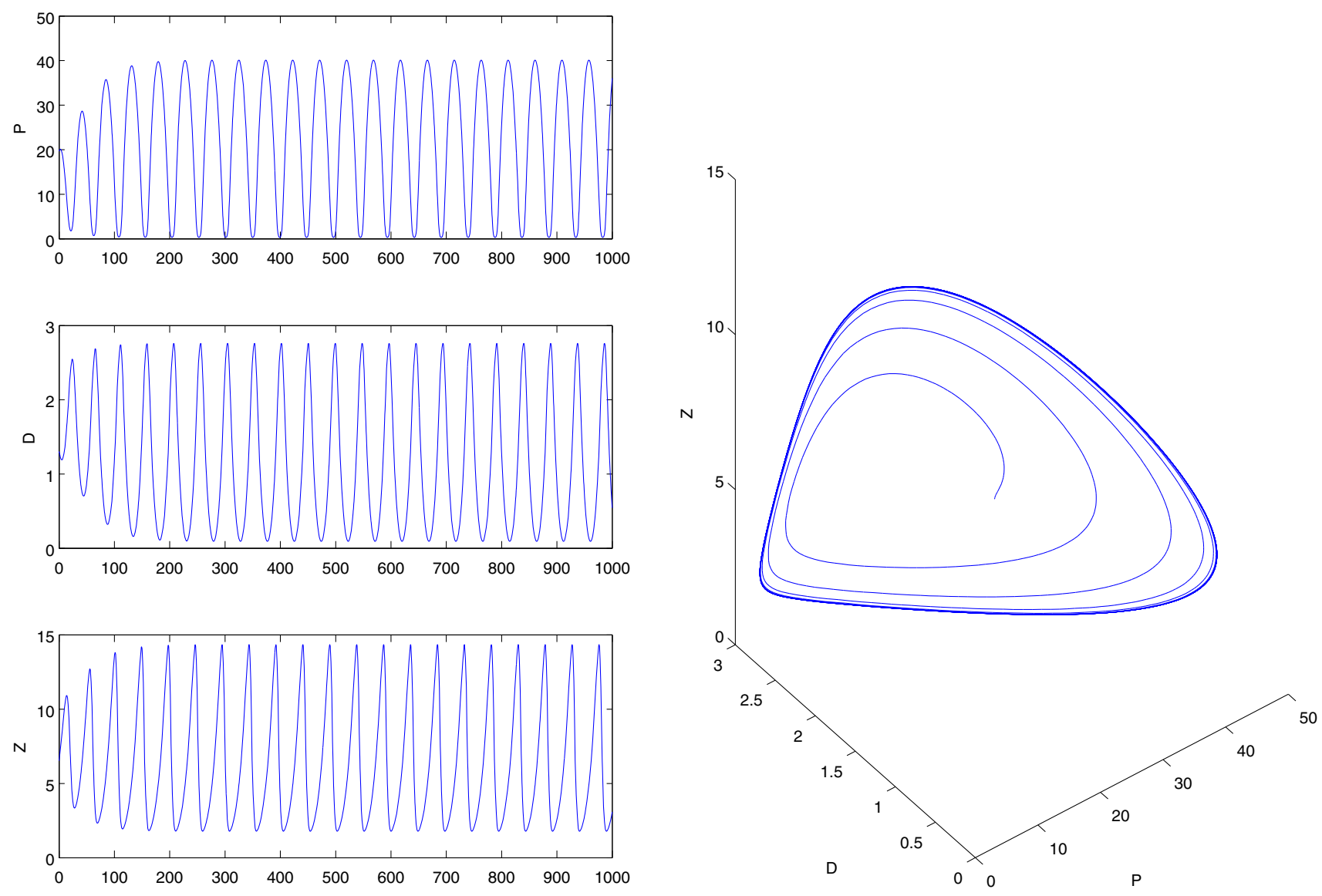

Fig. 3 The time series and phase space representation of three species around the endemic equilibrium, with $r=0.445533$

Now, we apply the normal form theory (Hassard et al. 1981), we can state the following theorem:

Theorem (Hassard et al. 1981) There is a Hopf bifurcation when $r$ passes through $r_{0}$ for system (7)-(9), and

(i) if $\mu_{2}>0(<0)$, the direction of bifurcation is supercritical (subcritical),

(ii) if $\beta_{2}<0(>0)$, the solutions of bifurcation periodic solutions are orbitally stable (unstable),

(iii) $\tau_{2}>0(<0)$, the periods of bifurcation periodic solutions increase (decrease). Here, we use $\mu_{2}, \beta_{2}$ and $\tau_{2}$ for more detail in Appendix-I.

\section{A numerical example}

Taking, the parametric values are $\delta_{1}=0.1, \beta=0.4$, $\beta_{1}=0.4, D_{0}=3, \alpha_{1}=0.18, \alpha_{2}=0.2, \delta_{2}=0.3, \eta=2.85$, $\zeta=0.2, a=3$ and $r=0.445533$. It follows from the results in "Direction and stability of the hopf bifurcation" that $\mu_{2}=0.00648118, \quad \beta_{2}=-0.00292106 \quad$ and $\tau_{2}=0 .+0.00508774 i$. In the light of Theorem, since $\mu_{2}>0$, the Hopf bifurcation is supercritical. Since, $\beta_{2}<0$, each individual periodic solution is stable and $\tau_{2}>0$, periods of bifurcating periodic solutions increase (see Figs. 2, 3).

\section{Model with diffusion}

We now consider the plankton dynamics with diffusion in system (1)-(3). In this case population densities will become space and time dependent, i.e., $P(t, x, y)$, $D(t, x, y)$ and $Z(t, x, y)$ are the population densities of phytoplankton, dissolved oxygen and zooplankton at time $t$ and space coordinate $(x, y)$. Then, our proposed model can stated by the following reaction diffusion system:

$$
\begin{aligned}
& \frac{\partial P}{\partial t}=\frac{r P}{\left(\zeta+D_{0}-D\right)}-\delta_{1} P-\frac{\beta P Z}{(P+a)}+D_{a} \nabla^{2} P, \\
& \frac{\partial D}{\partial t}=\eta\left(D_{0}-D\right)-\alpha_{1} P-\alpha_{2} Z+D_{b} \nabla^{2} D, \\
& \frac{\partial Z}{\partial t}=\frac{\beta_{1} P Z}{(P+a)}-\delta_{2} Z+D_{c} \nabla^{2} Z,
\end{aligned}
$$


$P(x, y, 0)>0, D(x, y, 0)>0, Z(x, y, 0)>0(x, y) \in \Omega$ and

$\frac{\partial P}{\partial n}=\frac{\partial D}{\partial n}=\frac{\partial Z}{\partial n}=0, \quad(x, y) \epsilon \partial \Omega, \quad t>0$,

where $\left(\nabla^{2}=\frac{\partial^{2}}{\partial x^{2}}+\frac{\partial^{2}}{\partial y^{2}}\right)$ is the Laplacian operator in twodimensional cartesian coordinate on a bounded domain $\Omega$ and $\mathrm{n}$ is the outward normal to $\partial \Omega . D_{a}, D_{b}$ and $D_{c}$ are diffusivity coefficients for the phytoplankton, dissolved oxygen and zooplankton population respectively and other parameters $\zeta, \beta, \beta_{1}, \eta, \alpha_{1}, \alpha_{2}, \delta_{1}, \delta_{2}$ are positive constant. The no-flux boundary condition is used.

Next, we will investigate the effect of diffusion on steady state of the reaction diffusion system. Obviously, the interior equilibrium point $E^{*}$ for the non-spatial system (1)-(3) is a spatially homogeneous steady-state for the reaction-diffusion system (11)-(13). We assume that $E^{*}$ is stable in the non-spatial system, which means that the spatially homogeneous equilibrium is stable with respect to spatially homogeneous with following perturbations:

$P(x, y, t)=P^{*}+\epsilon \exp \left(\left(k_{x}+k_{y}\right) i+\lambda_{k} t\right)$,

$D(x, y, t)=D^{*}+\eta \exp \left(\left(k_{x}+k_{y}\right) i+\lambda_{k} t\right)$,

$Z(x, y, t)=Z^{*}+\rho \exp \left(\left(k_{x}+k_{y}\right) i+\lambda_{k} t\right)$,

where $\epsilon, \eta$ and $\rho$ are chosen to be small and $k^{2}=\left(k_{x}^{2}+k_{y}^{2}\right)$ is the wave number. Substituting (16)-(18) into (11)-(13), we get the following characteristic equation of the linearized system around $E^{*}$ :

$\left|J_{k}-\lambda_{k} I_{3}\right|=0$

with

$J_{k}=\left[\begin{array}{ccc}a_{11}-D_{a} k^{2} & a_{12} & a_{13} \\ a_{21} & a_{22}-D_{b} k^{2} & a_{23} \\ a_{31} & a_{32} & a_{33}-D_{c} k^{2}\end{array}\right]$,

where $\quad a_{11}=\frac{\beta P^{*} Z^{*}}{\left(a+P^{*}\right)^{2}}, \quad a_{12}=\frac{r P^{*}}{\left(\zeta+D_{0}-D^{*}\right)^{2}}, \quad a_{13}=-\frac{\beta P^{*}}{a+P^{*}}$, $a_{21}=-\alpha_{1}, a_{22}=-\eta, a_{23}=-\alpha_{2}, a_{31}=\frac{a \beta_{1} Z^{*}}{\left(a+P^{*}\right)^{2}}, a_{32}=0$, $a_{33}=0$. The eigenvalues are the solutions of the characteristic equation,

$\lambda^{3}+p_{2} \lambda^{2}+p_{1} \lambda+p_{0}=0$

where

$p_{2}=\frac{1}{2}\left[\left(D_{a}+D_{b}+D_{c}\right) k^{2}+\eta-\frac{P Z \beta}{(a+P)^{2}}\right]$,

$$
\begin{aligned}
p_{1}= & \frac{1}{4}\left[k^{4}\left(D_{a} D_{b}+D_{a} D_{c}+D_{b} D_{c}\right)\right. \\
& +k^{2}\left(\eta\left(D_{a}+D_{c}\right)-\frac{P Z \beta}{(a+P)^{2}}\left(D_{b}+D_{c}\right)\right) \\
& \left.+\frac{P Z \beta}{(a+P)^{2}}\left(\frac{P \beta_{1}}{(a+P)}-\eta\right)+\frac{P r \alpha_{1}}{\left(\zeta+D_{0}-D\right)^{2}}\right], \\
p_{0}= & \frac{1}{8}\left[D_{a} D_{b} D_{c} k^{6}+k^{4}\left(D_{a} D_{b} \eta-\frac{D_{b} D_{c} P Z \beta}{(a+P)^{2}}\right)\right. \\
& +k^{2}\left(\frac{D_{b} P^{2} Z \beta \beta_{1}}{(a+P)^{3}}-\frac{D_{c} P Z \beta \eta}{(a+P)^{2}}+\frac{D_{c} P r \alpha_{1}}{\left(\zeta+D_{0}-D\right)^{2}}\right) \\
& \left.+\frac{P^{2} Z r \beta_{1} \alpha_{2}}{(a+P)^{2}\left(\zeta+D_{0}-D\right)^{2}}+\frac{P^{2} Z \beta \beta_{1} \eta}{(a+P)^{3}}\right] .
\end{aligned}
$$

According to the Routh-Hurwitz criterium, all the eigenvalues have negative real parts if and only if the following conditions hold:
(a) $\quad p_{2}>0$
(b) $\quad p_{0}>0$
(c) $Q=p_{0}-p_{1} p_{2}<0$.

This is clear from the invariants of the matrix and of its inverse matrix

$J_{k}^{-1}=\frac{1}{\operatorname{det}\left(J_{k}\right)}\left(\begin{array}{lll}M_{11} & M_{12} & M_{13} \\ M_{21} & M_{22} & M_{23} \\ M_{31} & M_{32} & M_{33}\end{array}\right)$,

where $\quad M_{11}=0, \quad M_{12}=0, \quad M_{13}=-\left(\frac{\alpha_{2} r P}{\left(\zeta+D_{0}-D\right)^{2}}+\frac{\eta \beta P}{(a+P)}\right)$, $M_{21}=-\frac{a \alpha_{2} \beta_{1} Z}{(a+P)^{2}}, M_{22}=\frac{a \beta \beta_{1} P Z}{(a+P)^{3}}, M_{23}=\frac{\alpha_{2} \beta P Z}{(a+P)^{2}}+\frac{\alpha_{1} \beta P}{a+P}, M_{31}=$ $\frac{\eta a \beta_{1} Z}{(a+P)^{2}}, M_{32}=\frac{a r \beta_{1} P Z}{(a+P)^{2}\left(\zeta+D_{0}-D\right)^{2}}, M_{33}=\frac{\alpha_{1} r P}{\left(\zeta+D_{0}-D\right)^{2}}-\frac{\eta \beta P Z}{(a+P)^{2}}$.

Here, matrix $M_{i j}$ is the adjunct of $J_{k}$.

It is obvious that these conditions possess a symmetry with respect to $J_{k}$ and $J_{k}^{-1}$. Contradiction of any one of the above conditions implies the existence of an eigenvalue with positive real part, hence instability. We obtain the following conditions of the steady-state stability (i.e., stability for any value of $\mathrm{k}$ ):

(i) All diagonal cofactors of matrix $J_{k}$ must be positive.

(ii) All diagonal elements of matrix $J_{k}$ must be negative.

The two above condition taken together are sufficient to ensure stability of a give steady state. It means that instability for some $k>0$ can only be observed if at least one of 
them is violated. Thus we arrive at the following necessary condition for the Turing instability (Baghel et al. 2012; Qian and Murray 2003):

(i) The largest diagonal element of matrix $J_{k}$ must be positive and/or (ii) the smallest diagonal cofactor of matrix $J_{k}$ must be negative.

By the Routh-Hurwitz criteria, instability takes place if and only if one of the conditions (24)-(26) is broken. We consider (25) for instability condition:

$$
\begin{aligned}
p_{0}(k)= & D_{a} D_{b} D_{c} k^{6}-\left(D_{a} D_{b} a_{33}+D_{b} D_{c} a_{11}+D_{a} D_{c} a_{22}\right) k^{4} \\
& +\left(D_{a} M_{11}+D_{b} M_{22}+D_{3} M_{33}\right) k^{2}-\operatorname{det} J
\end{aligned}
$$

According to Routh-Hurwitz criterium $p_{0}\left(k^{2}\right)<0$ is sufficient condition for matrix $J_{k}$ being unstable. Let us assume that $M_{33}<0$. If we choose $D_{a}=0, D_{b}=0$ and

$$
\begin{aligned}
p_{0}\left(k^{2}\right) & =D_{c} M_{33} k^{2}-\operatorname{det}\left(J_{k}\right) \\
& =-\left(\frac{P^{2} Z r \beta_{1} \alpha_{2}}{(a+P)^{2}\left(\zeta+D_{0}-D\right)^{2}}+\frac{P^{2} Z \beta \beta_{1} \eta}{(a+P)^{3}}\right)<0 .
\end{aligned}
$$

Hence, in this system diffusion-driven instability occur.

Now, we obtained the eigenvalues of the characteristic equation (20) numerically of the spatial system (11)-(13). Here, we choose some parametric values of $r=0.445532$, $a=3.0, \zeta=0.2, \delta_{1}=0.1, \beta=0.2, \quad \beta_{1}=0.4, D_{0}=3$, $\alpha_{1}=0.18, \alpha_{2}=0.2, \delta_{2}=0.3, \eta=2.085$. From (28), in this set of values $P_{0}\left(k^{2}\right)<0, \forall k>0$, (see Fig. 4).

\section{Spatiotemporal pattern formation}

It is well known that the analytical solution of the coupled reaction-diffusion system is not always possible. Hence, one has to use numerical simulations to solve them. In this section, the two-dimensional spatiotemporal system (11)(13) is solved numerically using a finite difference approximation method for the spatial derivatives. In order to avoid numerical artifacts steps of time and space have been chosen sufficiently small. Since we have chosen a closed environment, so all the numerical simulations use the zero-flux boundary conditions in a square habitat of size $100 \times 100$ and $200 \times 200$ and iterations are performed for different step sizes in time.

Now, we obtained that the spatial distributions of plankton dynamics in the time evaluation in Figs. 5, 6 and 7. By varying coupling parameters, we observe that if one parameter value changes then spatial structure changes over the time of the spatial system. In Figs. 5, 6 and 7, it has been observed well organized structures for the spatial distribution population also observed that as time $T$ increases from 100 to 1000 the density of different classes of population becomes uniform throughout the space. Finally, all these figures show the qualitative changes in spatial density distribution of the spatial system for the each species.

Also, we have shown the effect of the diffusivity coefficients in the system (11)-(13) and observed that the system is stabilized as diffusivity coefficients increases (see Figs. 8, 9).

\section{Higher order stability analysis}

In this section, we will study the stability of the higherorder spatiotemporal perturbation terms (Riaz et al. 2007). Without loss of generality our proposed system (11)-(13) can be described as three dimensional reaction-diffusion system as follows:

$$
\begin{aligned}
& u_{t}=f(u, v, w)+D_{a}\left(u_{x x}+u_{y y}\right), \\
& v_{t}=g(u, v, w)+D_{b}\left(v_{x x}+v_{y y}\right), \\
& w_{t}=h(u, v, w)+D_{c}\left(w_{x x}+w_{y y}\right),
\end{aligned}
$$

with initial distribution and no-flux boundary conditions of population within $2 \mathrm{D}$ closed domain. The non-zero equilibrium $E_{2}$ of the system (1)-(3) is a spatially homogeneous equilibrium for the system (29)-(31). We consider that in the temporal model $E^{*}=\left(u^{*}, v^{*}, w^{*}\right)$ is locally asymptotically stable and also taking the spatial perturbations in spatial system $u(t, x, y), v(t, x, y)$ and $w(t, x, y)$ around the steady states $u^{*}, v^{*}, w^{*}$ defined by $u=u^{*}+n(t, x, y)$, $v=v^{*}+p(t, x, y), w=w^{*}+m(t, x, y)$ and finally expanding the temporal part using Taylor series up to second order, we get following three expressions:

$$
\begin{aligned}
n_{t}= & f_{u} n+f_{v} p+f_{w} m+\frac{f_{u u}}{2} n^{2}+\frac{f_{v v}}{2} p^{2}+\frac{f_{w w}}{2} m^{2}+f_{u v} n p \\
& +f_{v w} p m+f_{u w} n m+D_{a}\left(n_{x x}+n_{y y}\right), \\
p_{t}= & g_{u} n+g_{v} p+g_{w} m+\frac{g_{u u}}{2} n^{2}+\frac{g_{v v}}{2} p^{2}+\frac{g_{w w}}{2} m^{2}+g_{u v} n p \\
& +g_{v w} p m+g_{u w} n m+D_{b}\left(p_{x x}+p_{y y}\right) . \\
m_{t}= & h_{u} n+h_{v} p+h_{w} m+\frac{h_{u u}}{2} n^{2}+\frac{h_{v v}}{2} p^{2}+\frac{h_{w w}}{2} m^{2}+h_{u v} n p \\
& +h_{v w} p m+h_{u w} n m+D_{c}\left(m_{x x}+m_{y y}\right) .
\end{aligned}
$$

Now, we taking particular periodic type of spatial perturbations:

$$
\begin{aligned}
n(t, x, y) & =n(t) \cos \left(k_{x} x\right) \cos \left(k_{y} y\right), \\
p(t, x, y) & =p(t) \cos \left(k_{x} x\right) \cos \left(k_{y} y\right), \\
m(t, x, y) & =m(t) \cos \left(k_{x} x\right) \cos \left(k_{y} y\right),
\end{aligned}
$$

with boundary conditions, we get the following three system of equations: 
(a)

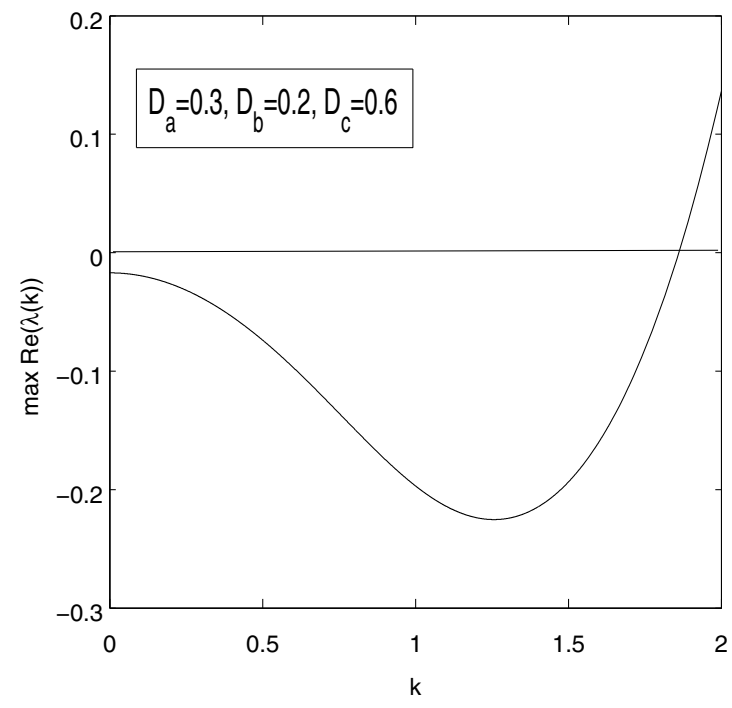

(c)

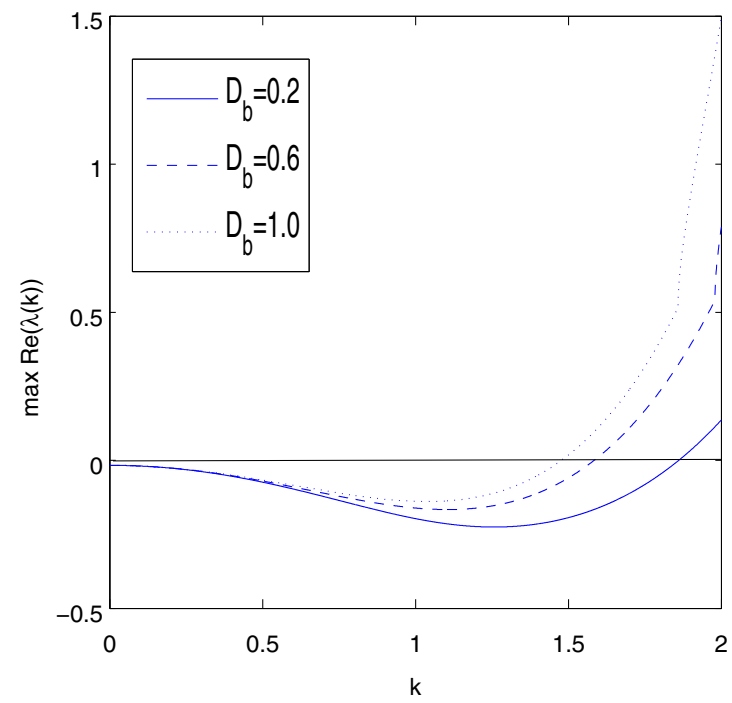

(b)

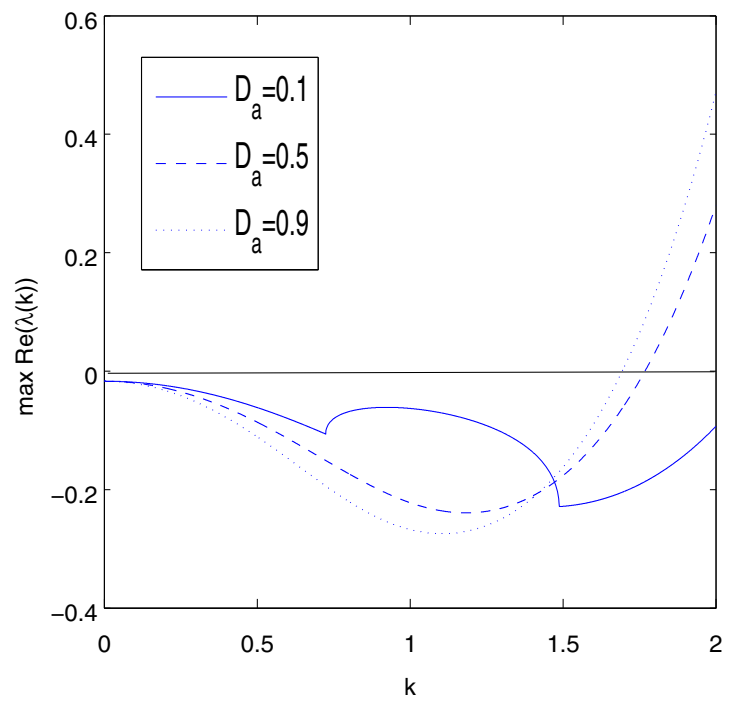

(d)

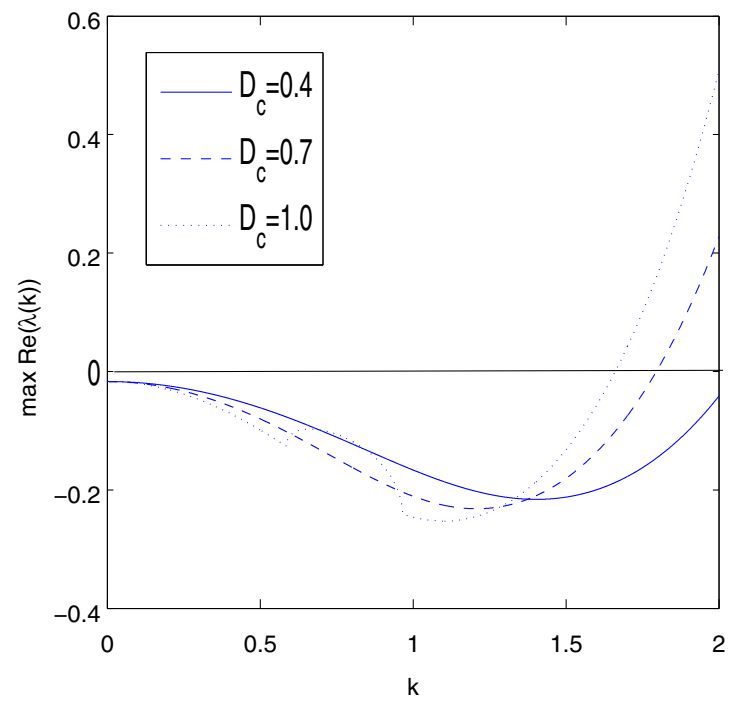

Fig. 4 Maximum $\operatorname{Re}(\lambda(k))$ against k. The other parametric values are given in text. Here, we can observe that diffusion driven instability in this system

$$
\begin{aligned}
n_{t}= & f_{u} n+f_{v} p+f_{w} m+\frac{f_{u u}}{2} n^{2}+\frac{f_{v v}}{2} p^{2}+\frac{f_{w w}}{2} m^{2}+f_{u v} n p \\
& +f_{v w} p m+f_{u w} n m-D_{a} k^{2} n, \\
p_{t}= & g_{u} n+g_{v} p+g_{w} m+\frac{g_{u u}}{2} n^{2}+\frac{g_{v v}}{2} p^{2}+\frac{g_{w w}}{2} m^{2}+g_{u v} n p \\
& +g_{v w} p m+g_{u w} n m-D_{b} k^{2} p .
\end{aligned}
$$

$m_{t}=h_{u} n+h_{v} p+h_{w} m+\frac{h_{u u}}{2} n^{2}+\frac{h_{v v}}{2} p^{2}+\frac{h_{w w}}{2} m^{2}+h_{u v} n p$

$$
+h_{v w} p m+h_{u w} n m-D_{c} k^{2} m \text {. }
$$

It is observed from above three equations that the increase or decrease of first-order perturbation terms depends upon the second-order perturbation terms.
Again, multiplying (35) by $2 \mathrm{n}$ and neglecting the contribution of third-order perturbation terms, we find the dynamical equation of $n^{2}$ in terms of second-order perturbation as

$$
\left(n^{2}\right)_{t}=2 f_{u} n^{2}+2 f_{v} n p+2 f_{w} n m-2 D_{a} k^{2} n^{2},
$$

and similarly, we can obtain the dynamical equations for the rest of the second-order perturbations as

$$
\begin{aligned}
& \left(p^{2}\right)_{t}=2 g_{u} n p+2 g_{v} p^{2}+2 g_{w} p m-2 D_{b} k^{2} p^{2}, \\
& \left(m^{2}\right)_{t}=2 h_{u} n m+2 h_{v} p m+2 h_{w} m^{2}-2 D_{c} k^{2} m^{2}, \\
& (n p)_{t}=g_{u} n^{2}+f_{v} p^{2}+\left(f_{u}+g_{v}\right) n p-k^{2}\left(D_{a}+D_{b}\right) n p,
\end{aligned}
$$


(a)

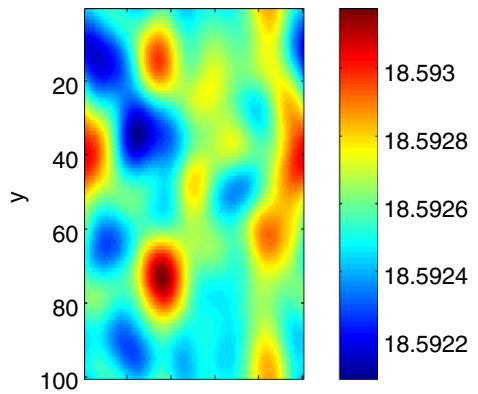

$20 \quad 40 \quad 60 \quad 80 \quad 100$

$\mathrm{x}$

(d)

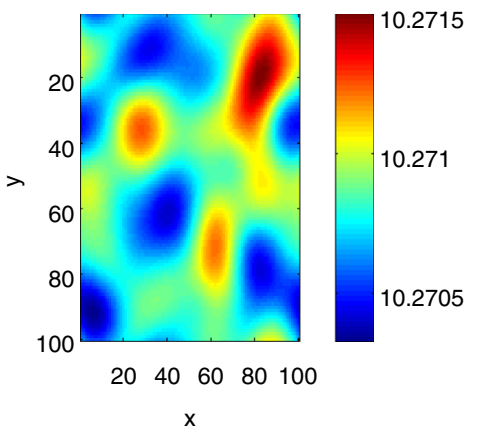

(g)

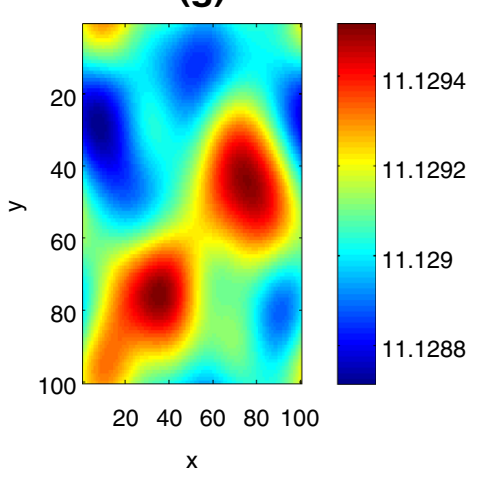

(b)

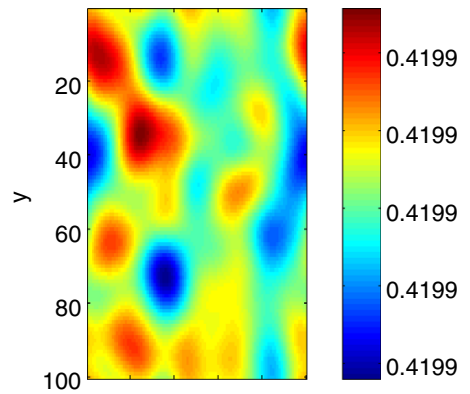

$20 \quad 40 \quad 60 \quad 80 \quad 100$

(e)

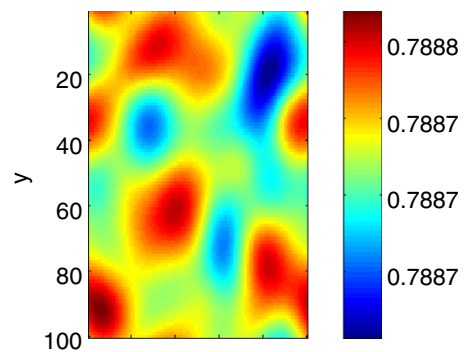

$204060 \quad 80100$

(h)

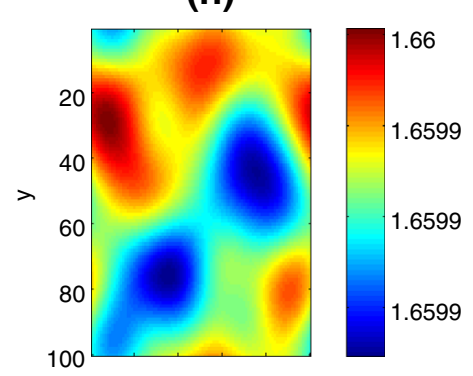

$204060 \quad 80100$ (c)

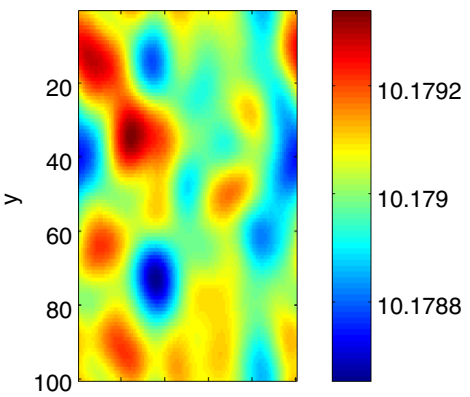

$2040 \quad 60 \quad 80100$

$\mathrm{x}$

(f)

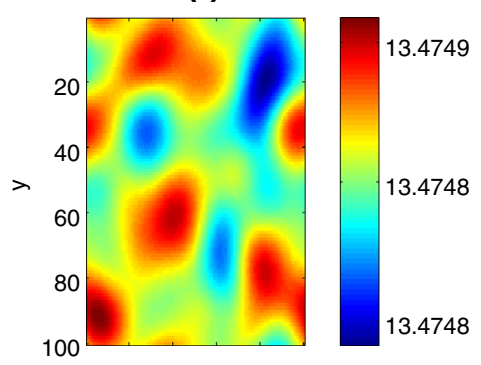

$204060 \quad 80100$

$\mathrm{x}$

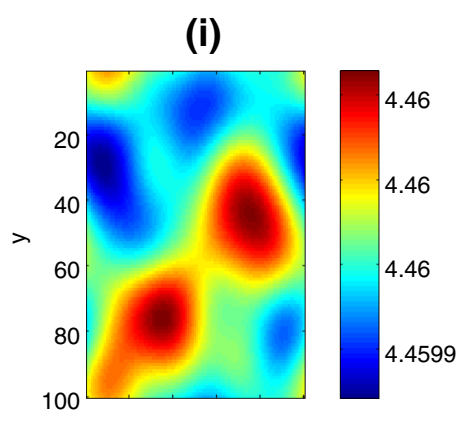

20406080100
Fig. 5 Spatial distribution of phytoplankton (first column), dissolved oxygen (second column) and zooplankton (third column) population density of the system (11)-(13). Spatial patterns are obtained with diffusivity coefficient $D_{a}=0.06, D_{b}=0.04, D_{c}=0.02$ at different time levels: for plot $T=300(a, b, c), T=500(d, e, f), T=$ $1000(g, h, i)$

$$
\begin{aligned}
(p m)_{t}= & h_{v} p^{2}+g_{w} m^{2}+g_{u} n m+h_{u} n p+\left(g_{v}+h_{w}\right) p m \\
& -k^{2}\left(D_{b}+D_{c}\right) p m, \\
(n m)_{t}= & h_{u} n^{2}+f_{w} m^{2}+f_{v} p m+h_{v} n p+\left(f_{u}+h_{w}\right) n m \\
& -k^{2}\left(D_{a}+D_{c}\right) n m .
\end{aligned}
$$

In the dynamical equations (35)-(43), we have truncated all third and higher-order terms in Taylor series expansion and also neglected all third and higher-order terms, it resulting us to a closed system of equations for $n, p, m, n^{2}, p^{2}, m^{2}, n$ $p, p m, n m$. Otherwise, it is impossible to avoid infinite hierarchy of dynamical equations for perturbation terms. Implication of the analysis for the system (11)-(13) can be justified with the perturbation terms up to order two with special choice of parameter values. Rewriting the dynamical equations (35)-(43) in matrix form as follows:

$\frac{d X}{d t}=A X$

where, $X=\left[n, p, m, n^{2}, p^{2}, m^{2}, n p, p m, n m\right]^{T}$ and 
(a)

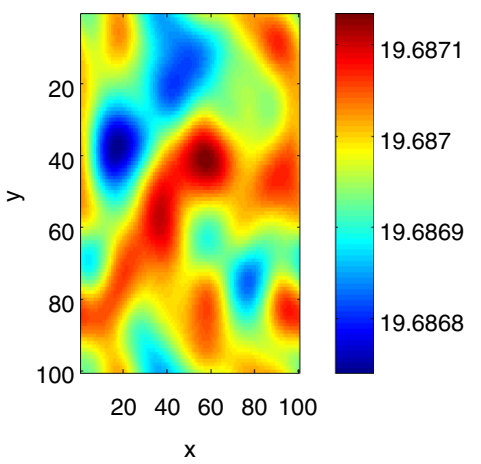

(d)

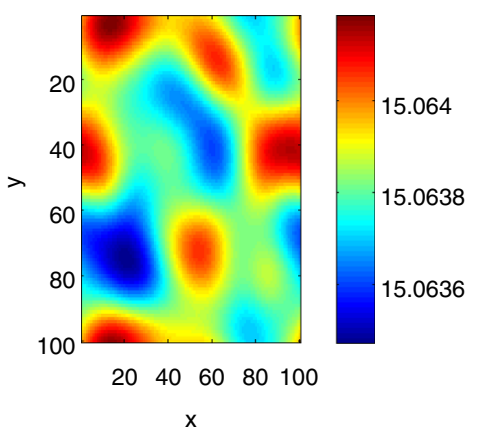

(g)

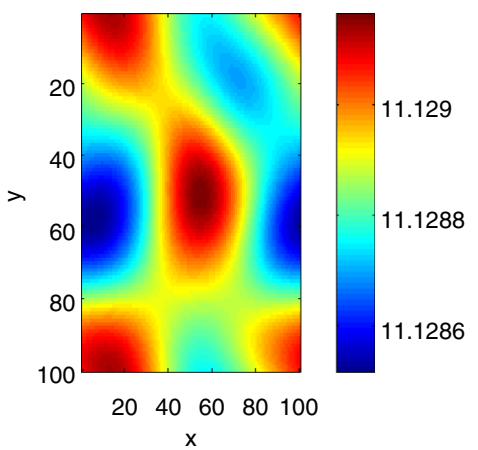

(b)

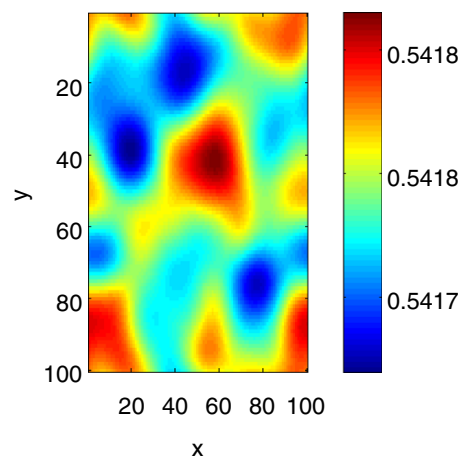

(e)

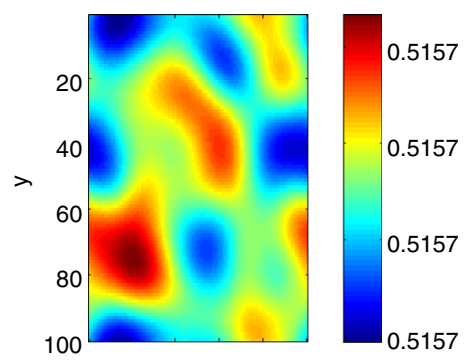

$20 \quad 40 \quad 60 \quad 80 \quad 100$

(h)

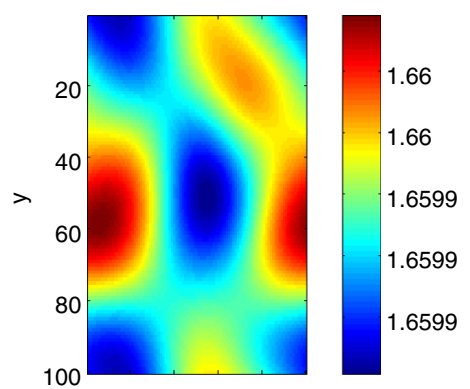

$2040 \quad 60 \quad 80100$ (c)

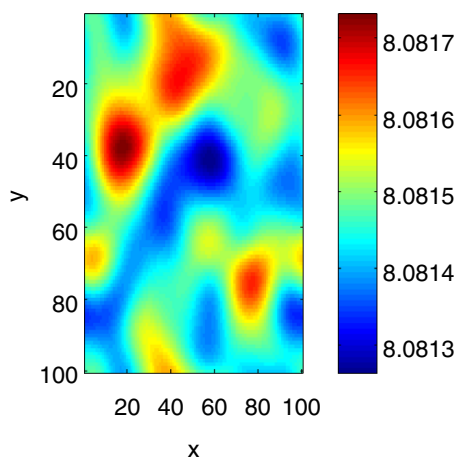

(f)

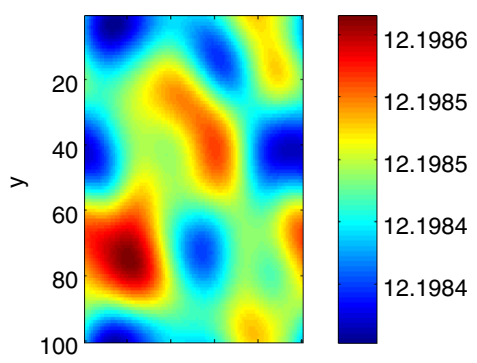

$20 \quad 40 \quad 60 \quad 80 \quad 100$

(i)

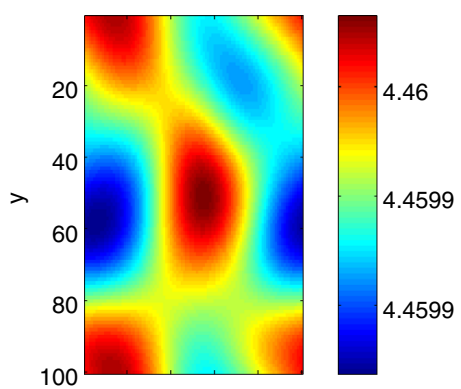

20406080100
Fig. 6 Spatial distribution of phytoplankton (first column), dissolved oxygen (second column) and zooplankton (third column) population density of the system (11)-(13). Spatial patterns are obtained with diffusivity coefficient $D_{a}=0.03, D_{b}=0.02, D_{c}=0.1$ at different time levels: for plot $\mathrm{T}=200(\mathrm{a}, \mathrm{b}, \mathrm{c}), \mathrm{T}=400(\mathrm{~d}, \mathrm{e}, \mathrm{f}), \mathrm{T}=$ $1000(\mathrm{~g}, \mathrm{~h}, \mathrm{i})$
$A=\left[\begin{array}{ccccccccc}a_{11} & f_{v} & f_{w} & \frac{f_{u u}}{2} & \frac{f_{v v}}{2} & \frac{f_{w w}}{2} & f_{u v} & f_{v w} & f_{u w} \\ g_{u} & a_{22} & g_{w} & \frac{g_{u u}}{2} & \frac{g_{v v}}{2} & \frac{f_{w w}}{2} & g_{u v} & g_{v w} & g_{u w} \\ h_{u} & h_{v} & a_{33} & \frac{h_{u u}}{2} & \frac{h_{v v}}{2} & \frac{h_{w w}}{2} & h_{u v} & h_{v w} & h_{u w} \\ 0 & 0 & 0 & a_{44} & 0 & 0 & 2 f_{v} & 0 & 2 f_{w} \\ 0 & 0 & 0 & 0 & a_{55} & 0 & 2 g_{u} & 2 g_{w} & 0 \\ 0 & 0 & 0 & 0 & 0 & a_{66} & 0 & 2 h_{v} & 2 h_{u} \\ 0 & 0 & 0 & g_{u} & f_{v} & 0 & a_{77} & 0 & 0 \\ 0 & 0 & 0 & 0 & h_{v} & g_{w} & h_{u} & a_{88} & g_{u} \\ 0 & 0 & 0 & h_{u} & 0 & f_{w} & h_{v} & f_{v} & a_{99}\end{array}\right]$, with $a_{11}=f_{u}-D_{a} k^{2}, a_{22}=g_{v}-D_{b} k^{2}, a_{33}=h_{w}-D_{c} k^{2}$, $a_{44}=2\left(f_{u}-D_{a} k^{2}\right), \quad a_{55}=2\left(g_{v}-D_{b} k^{2}\right), \quad a_{66}=2\left(h_{w}-\right.$ $\left.D_{c} k^{2}\right), \quad a_{77}=f_{u}+g_{v}-k^{2}\left(D_{a}+D_{b}\right), \quad a_{88}=g_{v}+h_{w}-k^{2}$ $\left(D_{b}+D_{c}\right), a_{99}=f_{u}+h_{w}-k^{2}\left(D_{a}+D_{c}\right)$.

Choosing the solution of the system of (44) in the form $X(t) \sim e^{\lambda t}$, we can get the characteristic equation for the matrix A

$\left|A-\lambda I_{5}\right|=0$,

and the roots of (45) are $\lambda \equiv \lambda(k)$, which are eigenvalues of A. For the instability condition requires at least one of the 
(a)

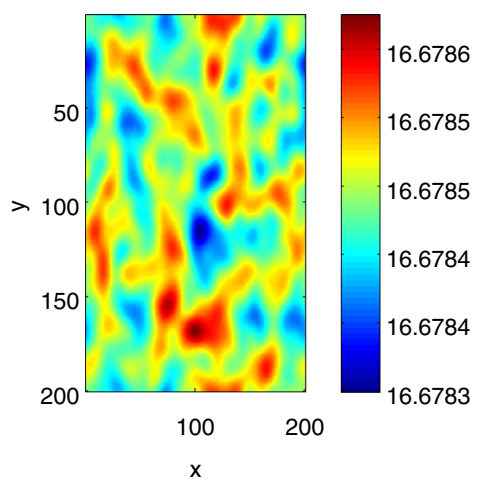

(d)

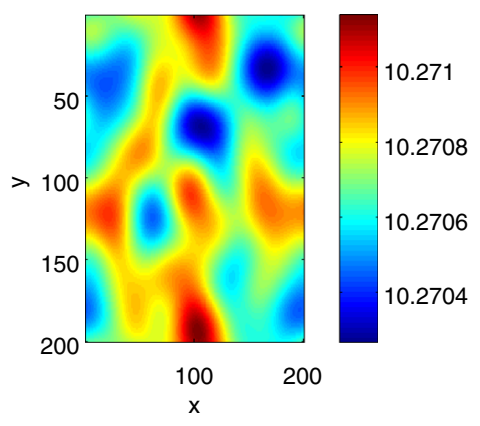

(g)

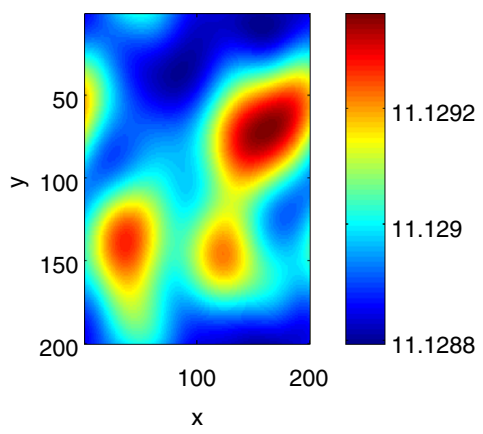

(b)

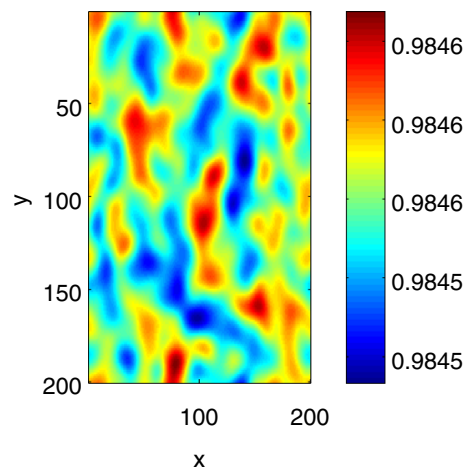

(e)

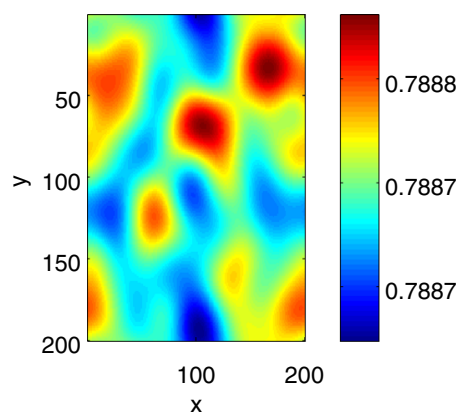

(h)

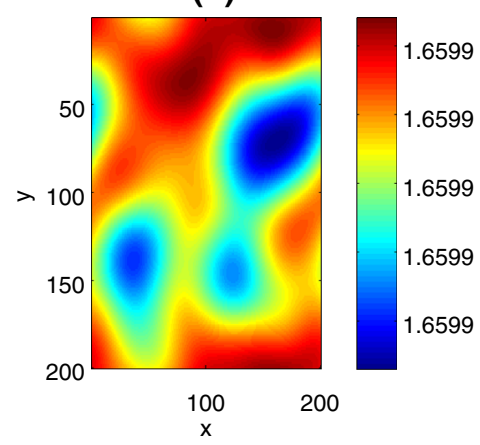

(c)

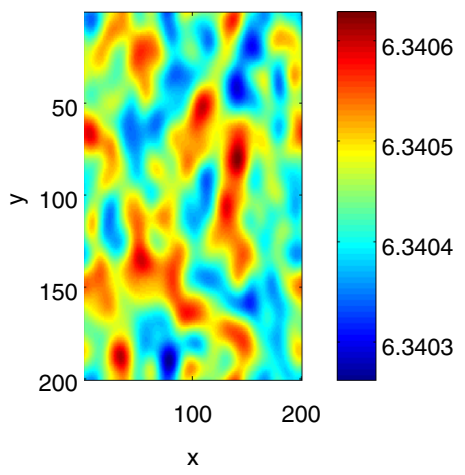

(f)

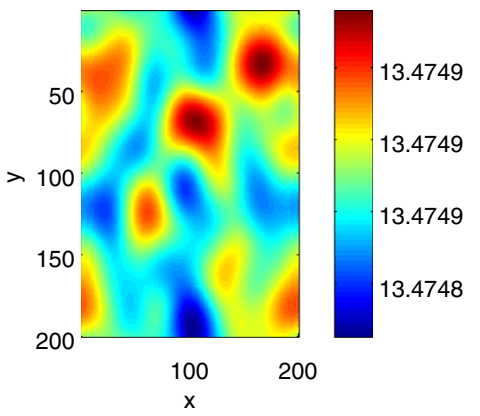

(i)

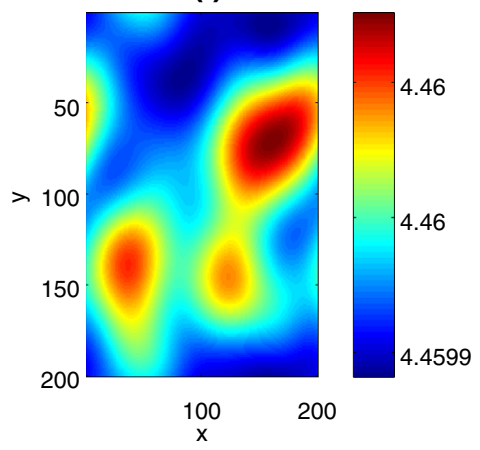

Fig. 7 Spatial distribution of phytoplankton (first column), dissolved oxygen (second column) and zooplankton (third column) population density of the system (11)-(13). Spatial patterns are obtained with diffusivity coefficient $D_{a}=0.1, D_{b}=0.2, D_{c}=0.03$ at different time levels: for plot $\mathrm{T}=100(\mathrm{a}, \mathrm{b}, \mathrm{c}), \mathrm{T}=500(\mathrm{~d}, \mathrm{e}, \mathrm{f}), \mathrm{T}=1000(\mathrm{~g}, \mathrm{~h}, \mathrm{i})$

values of $D_{c}=0.5,0.9,2.0,6.0$. In this case, the interior equilibrium point $\left(P^{*}, D^{*}, Z^{*}\right)=(9,1.67182,5.74631)$ is locally asymptotically stable for the temporal model (1)(3). For finding the possibility that one eigenvalue of the system (11)-(13) having positive real part, we have plotted largest $\operatorname{Re}\{\lambda(k)\} \equiv$ linear obtained by solving (20) along with largest $\operatorname{Re}\{\lambda(k)\} \equiv$ higher order computed numerically for the characteristic equation (45) for a range of wavelengths values of $\mathrm{k}$, shown in Fig. 10. It is observed that in the case of linear the $\max \operatorname{Re}\{\lambda(k)\}$ is positive after certain value of $k$, and in higher order $\max \operatorname{Re}\{\lambda(k)\}<0$, for 
(a)

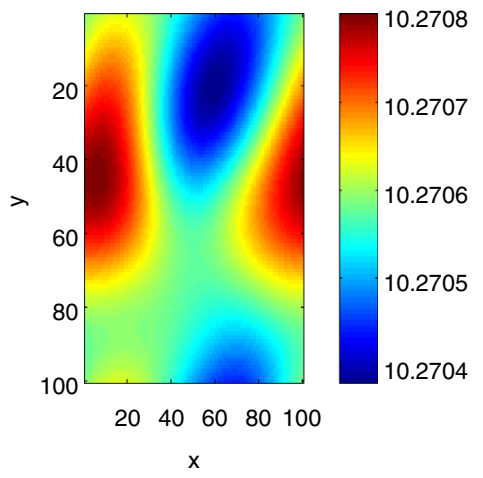

(d)

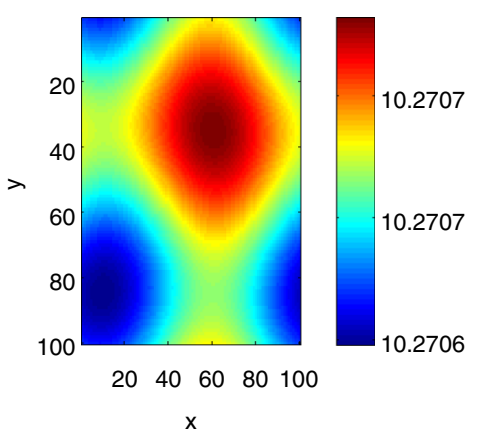

(g)

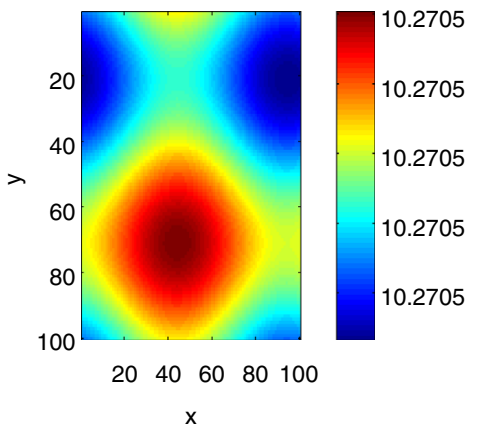

(b)

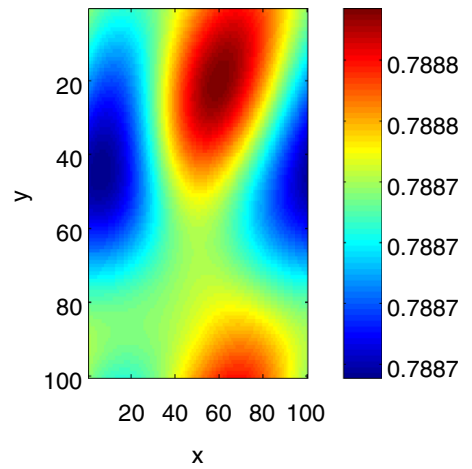

(e)

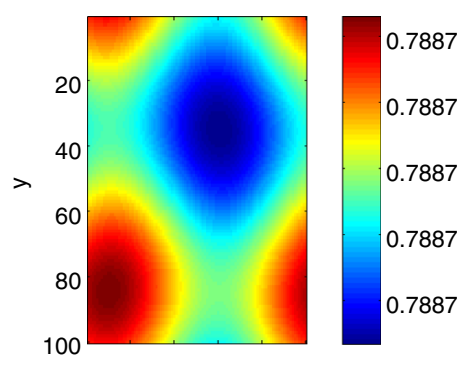

$20 \quad 40 \quad 60 \quad 80 \quad 100$

(h)

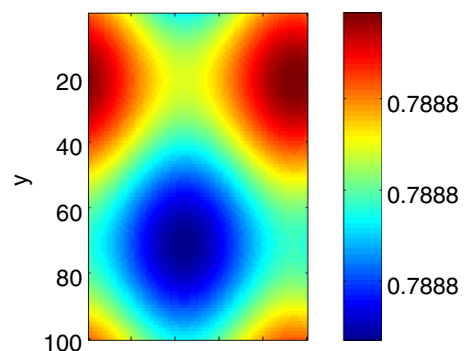

$20 \quad 40 \quad 60 \quad 80 \quad 100$ (c)

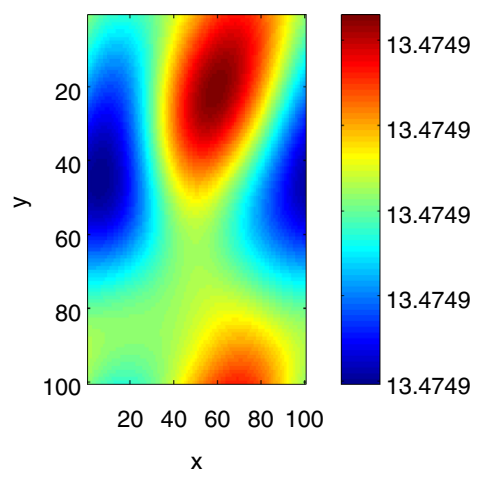

(f)

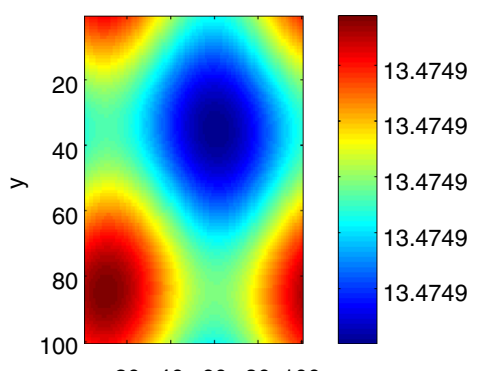

$20 \quad 40 \quad 60 \quad 80100$

(i)

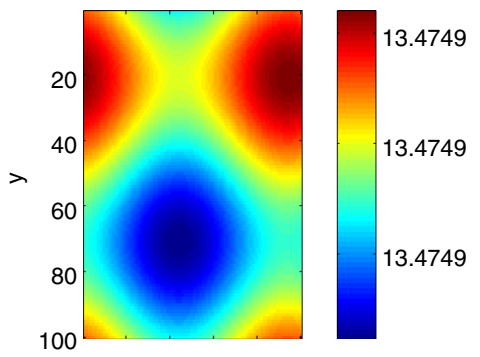

$2040 \quad 60 \quad 80 \quad 100$
Fig. 8 Spatial distribution of phytoplankton density (first column), dissolved oxygen density (second column) and zooplankton density (third column) of the spatial system (11)-(13). Spatial patterns are

all values of $k$. These results ensure that the linearly unstable system may become stable in higher order.

\section{Conclusions}

In this paper, we have studied a three dimensional plankton dynamics with and without diffusion. We have obtained the local stability and existence of Hopf-bifurcation with respect to $r$ as a bifurcation parameter (i.e., $r$ obtained at fixed time $T=500$ with diffusivity coefficients $D_{a}=0.3$, $D_{b}=0.2$ and for different values of $D_{c}$ : in $D_{c}=0.06(a, b, c)$, $D_{c}=0.6(d, e, f), D_{c}=2.0(g, h, i)$

the growth rate of phytoplankton due to the up-taking of dissolved oxygen) of the temporal system. If $r$, crosses its threshold value, i.e., $r=r_{0}$, then phytoplankton, dissolved oxygen and zooplankton population start oscillating around the endemic equilibrium. The above result has been shown numerically in Figs. 2, 3 for different values of $r$. Also we have obtained analytically the stability of the bifurcating solution of the temporal system. Furthermore, it has been established analytically as well as numerically that the diffusion driven instability occurs in 
(a)

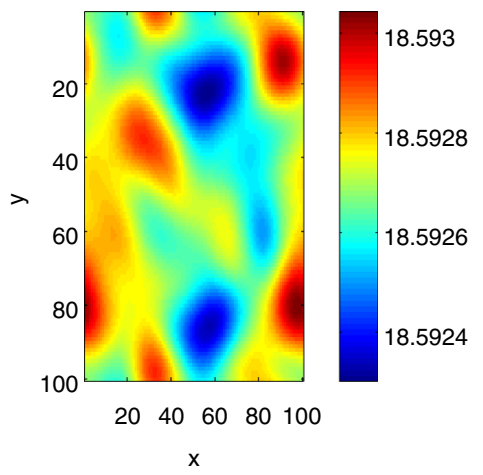

(d)

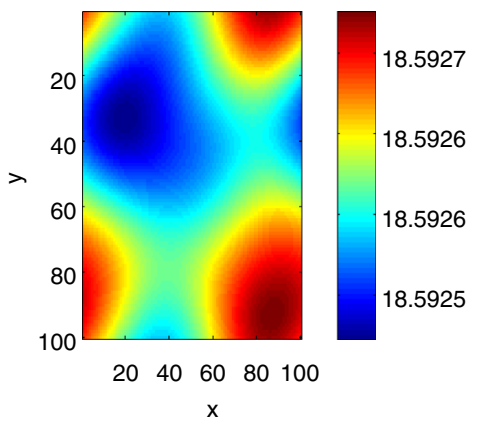

(g)

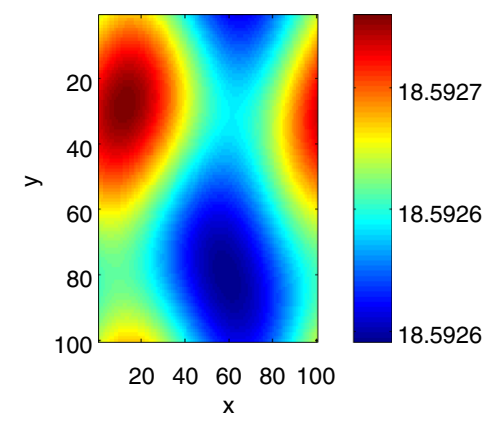

(b)

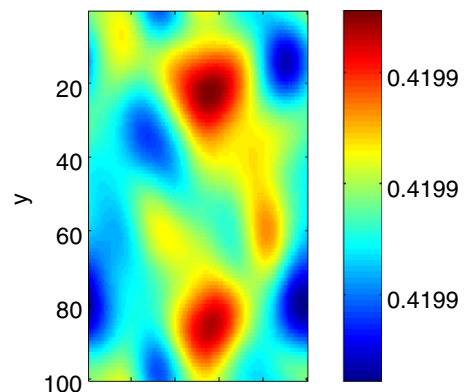

$20 \quad 40 \quad 60 \quad 80100$

(e)

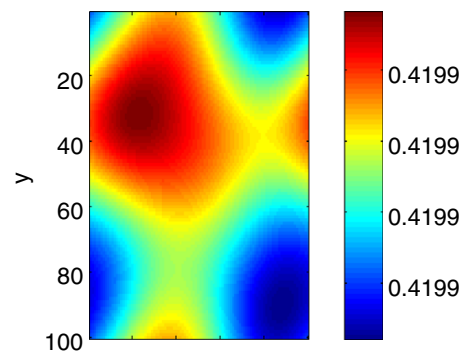

$204060 \quad 80100$

$\mathrm{x}$

(h)

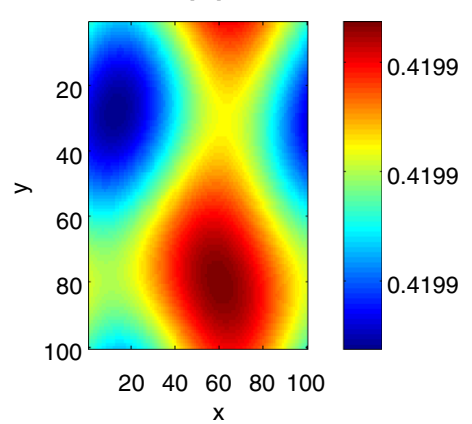

(c)

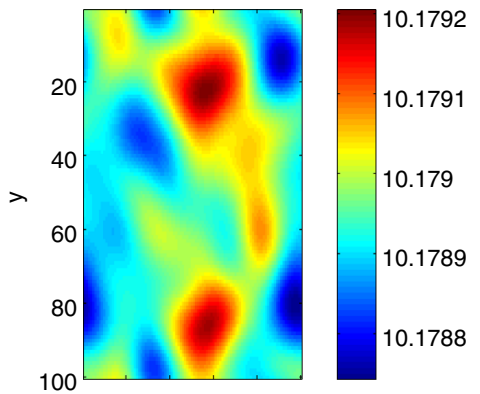

$2040 \quad 60 \quad 80100$

$\mathrm{X}$

(f)

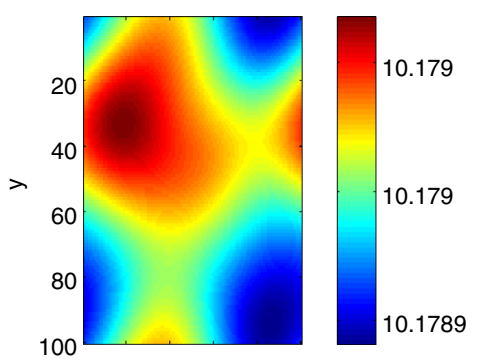

20406080100

(i)

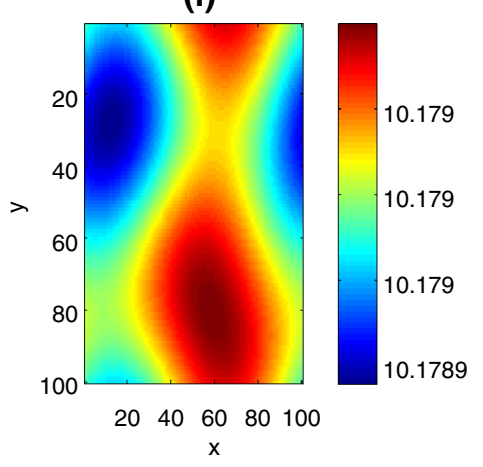

Fig. 9 Spatial distribution of phytoplankton density (first column), dissolved oxygen density (second column) and zooplankton density (third column) of the spatial system (11)-(13). Spatial patterns are obtained at fixed time $T=300$ with diffusivity coefficient $D_{b}=0.02$, $D_{c}=0.02$ and for different values of $D_{a}$ : in $D_{a}=0.1(a, b, c)$, $D_{a}=0.6(d, e, f), D_{a}=1.0(g, h, i)$ the spatial system (see Fig. 4). We have obtained the nature of spatial patterns with respect to time (see Figs. 5, $6,7)$. Also, we have shown the effect of diffusion on the spatiotemporal system in two dimensional case and it is shown that sufficiently large diffusivity coefficients are stabilized the system (see Figs. 8, 9). Further from higherorder stability analysis, and have observed that stability behavior remains unchanged for second order (see Fig. 10). Our results have suggested that modeling using reaction-diffusion equation is one of the appropriate tool 
(a)

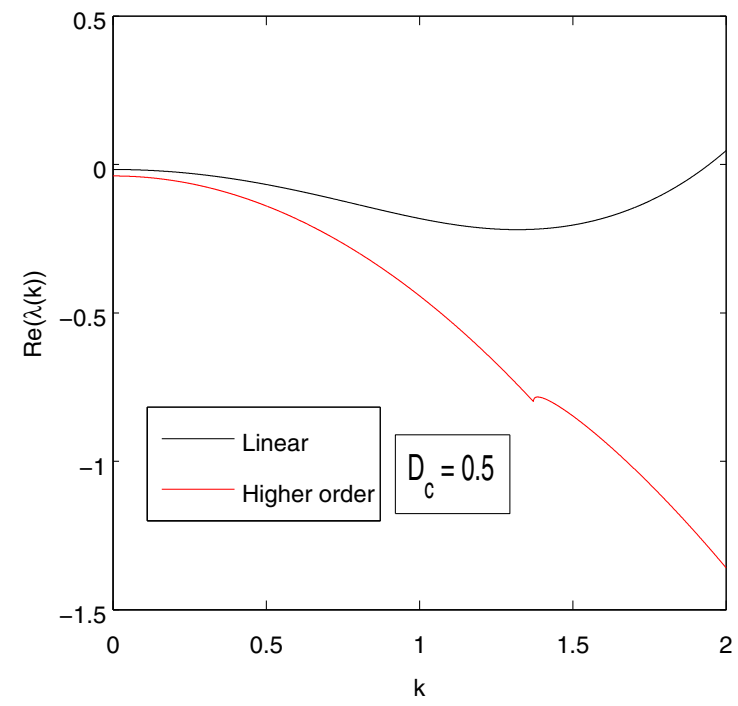

(c)

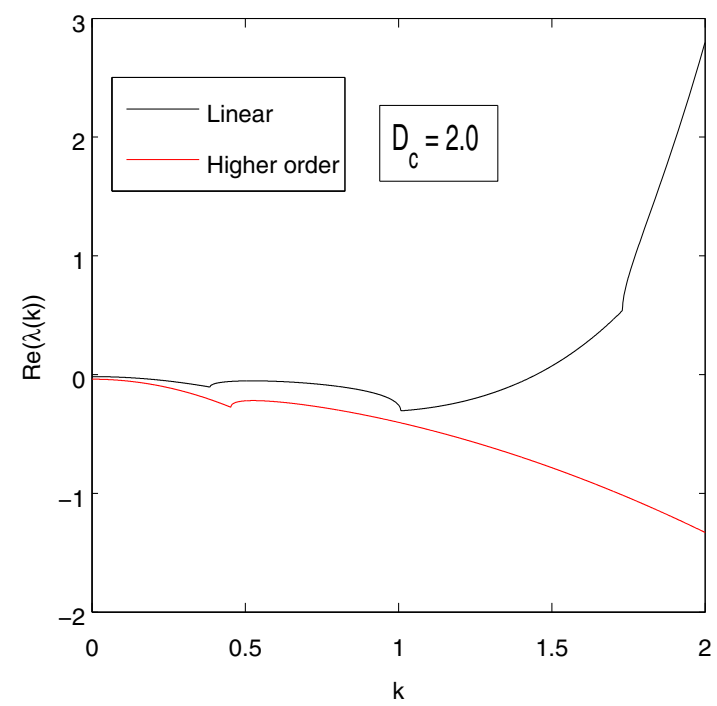

(b)

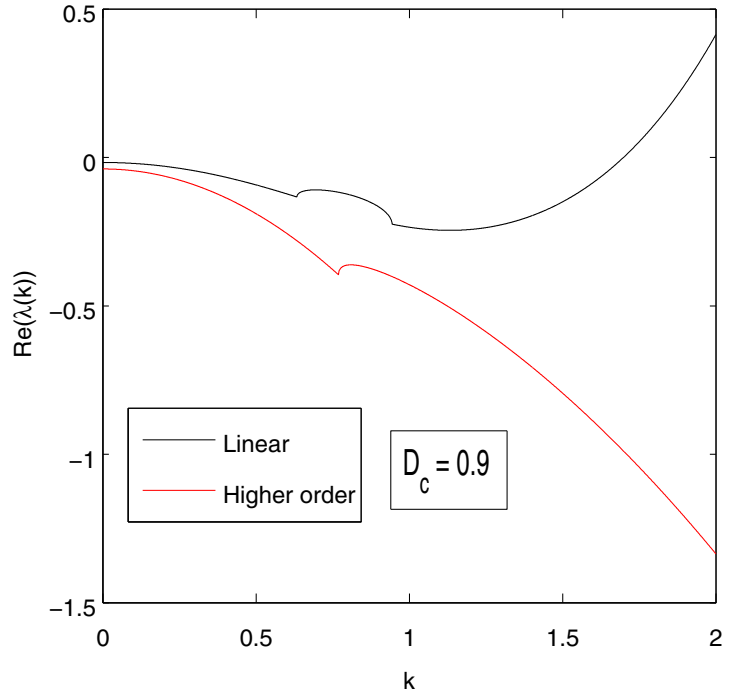

(d)

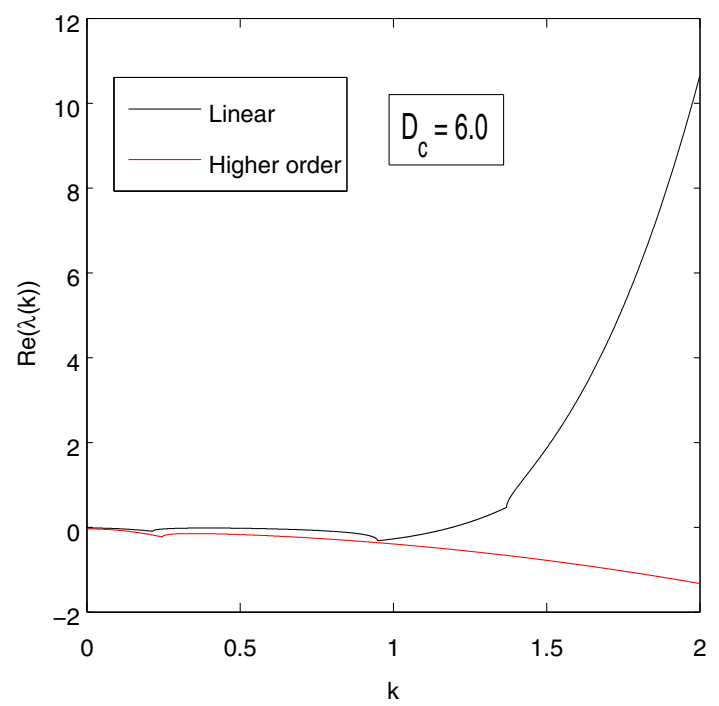

Fig. 10 Maximum of $\operatorname{Re}(\lambda(k))$ plots for (black line) linear and (red line) non-linear system versus $\mathrm{k}$. The parametric values are given in the text

for investigating fundamental mechanisms of complexity for the spatiotemporal dynamics. It may help us for better understanding of the plankton dynamics in a real environment.

\section{References}

Agusti S, Duarte C, Kalff J (1987) Algal cell size and the maximum density and biomass of phytoplankton. Limnol Oceangr 32:983-986

Baghel RS, Dhar J (2014) Pattern formation in three species food web model in spatiotemporal domain with beddingtondeangelis functional response. Nonlinear Anal Model Control 19:155-171
Baghel RS, Dhar J, Jain R (2011) Analysis of a spatiotemporal phytoplankton dynamics: higher order stability and pattern formation. World Acad Sci Eng Technol 60:1406-1412

Baghel RS, Dhar J, Jain R (2012) Bifurcaion and spatial pattern formation in spreading of disease with incubation period in a phytoplankton dynamics. Electron J Differ Equ 21:1-12

Baghel RS, Dhar J, Jain R (2012) Chaos and spatial pattern formation in phytoplankton dynamics. Elixir Appl Math 45:8023-8026

Baghel RS, Dhar J, Jain R (2012) Higher order stability analysis of a spatial phytoplankton dynamics: bifurcation, chaos and pattern formation. Int J Math Model Simul Appl 5:113-127

Bratbak G, Levasseur M, Michand S, Cantain G, Fernandez E, Heldal M (1995) Viral activity in relation to emiliania huxleyi bloom, a mechanism of dmsp release. Marine Prog Ser 128:133-142

Cantrell R, Cosner C (2004) Spatial ecology via reaction-diffusion equations. Wiley, New York 
Dhar J, Baghel RS, Sharma AK (2012) Role of instant nutrient replenishment on plankton dynamicswith diffusionin a closed system: a pattern formation. Appl Math Comput 218(17):8925-8936

Edward A, Brindley J (1996) Oscillatory behaviour in a three component plankton population model. Dyn Stab Syst 11:347-370

Egg J, Heimdal B (1994) Bloom of phytoplankton emiliania huxleyi. Effect of nutrient supply in different n:p ratio. Sarsia 79:327-347

Hassard B, Kazarinoff N, Wan Y (1981) Theory and application of hopf bifurcation. Cambridge University Presss, Cambridge

Holling C (1959) Some characteristics of simple types of predation and parasitism. Can Entomol 91:385-398

Huppert A, Blasius B, Olinky R, Stone L (2005) A model for seasonal phytoplankton blooms. J Theor Biol 236(3):276-290

Khare S, Kumar SSC (2013) Modelling effect of the depleting dissolved oxygen on the existence of interacting planktonic population. Elixir Appl Math 55:12739-12742

Mathews L, Brindley J (1996) Patchiness in plankton population. Dyn Stab Syst 12:39-59

Medvinsky A, Petrovskii S, Tikhonova I, Malchow H (2002) Spatiotemporal complexity of plankton and fish dynamic. SIAM Rev 44:311-370

Misra AK (2010) Modeling the depletion of dissolved oxygen in a lake due to submerged macrophytes. Nonlinear Anal Model Control 15(2): 185198

Murray J (2003) Mathematical biology II: spatial models and biomedical applications, 3rd edn. Springer, New York

Naik VK, Manjapp S (2010) Prediction of dissolved oxygen through mathematical modeling. Int J Environ Res 4(1):153-160

Petrovskii S, Malchow H (1999) A minimal model of pattern formation in a prey predator system. Math Comput Model 29:49-63
Petrovskii SV, Malchow H (2001) Wave of chaos: new mechanism of pattern formation in spatio-temporal population dynamic. Theor Population Biol 59:157-174

Pitchford J, Brindley J (1998) Intratrophic predation in simple predator-prey models. Bull Math Biol 60:511-524

Qian H, Murray JD (2003) A simple method of parameter space determination for diffusion-driven instability with three species. Appl Math Lett 14:405-411

Riaz SS, Sharma R, Bhattacharya SP, Ray DS (2007) Instability and pattern formation in reaction-diffusion systems: a higher order analysis. J Chem Phys 126:064,503

Richardson K, Heilmann JP (1995) Primary production in the kattegat; past and present. Ophelia 41:317-328

Ruan S (1993) Persistence and coexistence in zooplankton-phytoplankton-nutrient models with instantaneous nutrient recycling. J Math Biol 31(6):633-654

Sarkar RR, Malchow H (2005) Nutrients and toxin producing phytoplankton control algal blooms a spatio-temporal study in a noisy environment. J Biosci 30(5):749760

Shuklaa JB, Misrac AKCP (2008) Mathematical modeling and analysis of the depletion of dissolved oxygen in eutrophied water bodies affected by organic pollutants. Nonlinear Anal Real World Appl 9:18511865

Truscott J, Brindley J (1994) Ocean plankton population as excitable media. Bull Math Biol 56:991-998

Yi F, Wei J, Shi J (2009) Bifurcation and spatiotemporal patterns in a homogeneous diffusive predator-prey system. J Differ Equ 246:1944-1977

Zhang L, Chan C, Zhang F (2004) Development of water quality model in the satilla river estuary. Ga Ecol Model 78:457-482 